\title{
Development of pre-clinical and clinical models to predict the efficacy and safety of vaginal microbicides Betsy C Herold*
}

Address: Mount Sinai School of Medicine, New York, New York, 10029, USA

* Corresponding author

from 2006 International Meeting of The Institute of Human Virology

Baltimore, USA. 17-2I November, 2006

Published: 21 December 2006

Retrovirology 2006, 3(Suppl I):S53 doi:10.II86/I742-4690-3-SI-S53

(C) 2006 Herold; licensee BioMed Central Ltd.

HIV, genital herpes, and other sexually transmitted infections are a critical national and global health priority requiring the rapid development of safe and effective control methods. While condoms prevent HIV transmission, social acceptance is a major barrier and consistent use is difficult to achieve. Although there have been great strides in vaccine development, a fully protective vaccine unlikely to be available in the near future Thus, the development of vaginal microbicides has emerged as a priority in the effort to control the AIDS epidemic. An optimal microbicide should protect against infection but must also be safe, without adversely affecting the mucosal environment, including mediators of host defense. Over sixty candidate microbicides are in development and several are currently being tested in Phase III clinical trials. However, critical gaps in the preclinical and clinical development of microbicides include the identification of optimal assays to predict efficacy and safety. Identification of in vitro, animal model or pilot clinical assays that could serve as surrogate markers to predict the extent of antimicrobial activity and the safety of microbicides prior to embarking on largescale clinical trials are urgently needed. This will require a greater understanding of mucosal immunity and the viral and host factors that contribute to infection in the female genital tract. 